
\title{
Study of Content Based Image Retrieval Techniques
}

\author{
Rakhee $\mathbf{M}^{1}$, Indu M T ${ }^{2}$, Baiju Karun ${ }^{3}$ \\ ${ }^{1,2}$ Assistant Professor, Department of CSE, Muthoot Institute of Technology and Science, Varikoli, Ernakulam, Kerala, India \\ ${ }^{3}$ Associate Professor, Department of CSE, Muthoot Institute of Technology and Science, Varikoli, Ernakulam, Kerala, India
}

\begin{abstract}
Content-based image retrieval (CBIR) aims at retrieving images from a large database based on similarities in their features to a query image given by the user. It avoids the use of textual descriptions and instead retrieves images based on features like texture, colors, shapes etc. This involves the application of computer vision techniques to image retrieval problem. In this paper we present different techniques for content based image retrieval.
\end{abstract}

Keywords: Image Retrieval, Walshlet pyramid, relevance feedback, image tiling

\section{Introduction}

An image retrieval system is a system that allows its users to browse, search and retrieve images from a large collection of images. To search for images, a user provides query in the form of keywords or image file and the system will return images "similar" to the query. Meta tags, color distribution in images, region/shape attributes, etc can be the similarity used for search criteria. Image retrieval can be used for a variety of applications like Architectural and engineering design, Art collections, Crime prevention, Geographical information and remote sensing systems, Medical diagnosis, Military, Photograph archives, Retail catalogs, Face Finding and Textiles Industry.

Two common methods of image retrieval are image meta search and content based image retrieval. The first type utilize some method of adding metadata such as captions, tags, keywords, or descriptions to the images so that retrieval can be performed over the annotated words. The second type, called Content-based image retrieval (CBIR) involves the application of computer vision to the image retrieval as shown in Fig. 1.

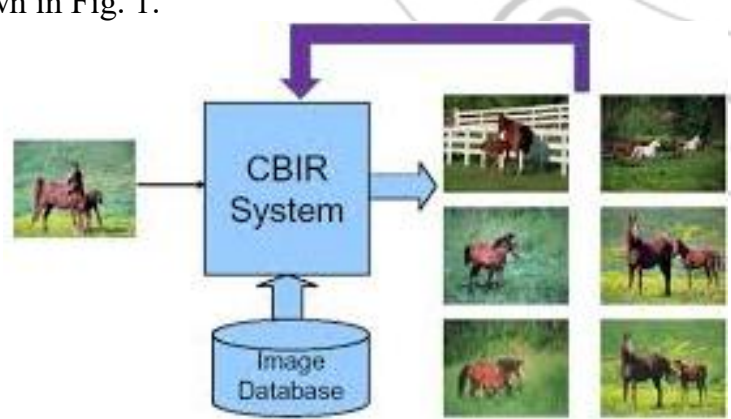

Figure 1: Content Based Image Retrieval System

The idea of content-based image retrieval originated in 1992, by scientist T.Kato. He experimented about the retrieval of images from a small database by using their visual content. Pattern recognition, signal processing, linear systems and machine vision areas are used to create the techniques, tools and algorithms that are used in CBIR. CBIR avoids the use of textual descriptions and instead retrieves images based on features like texture, colors, shapes etc. A CBIR system generally works as shown in Fig. 2. Features are extracted from an image database and stored as feature database. The features of the input query image are collected and some similarity measures are calculated between the features of query image and features in feature database. Then final results are retrieved and displayed.

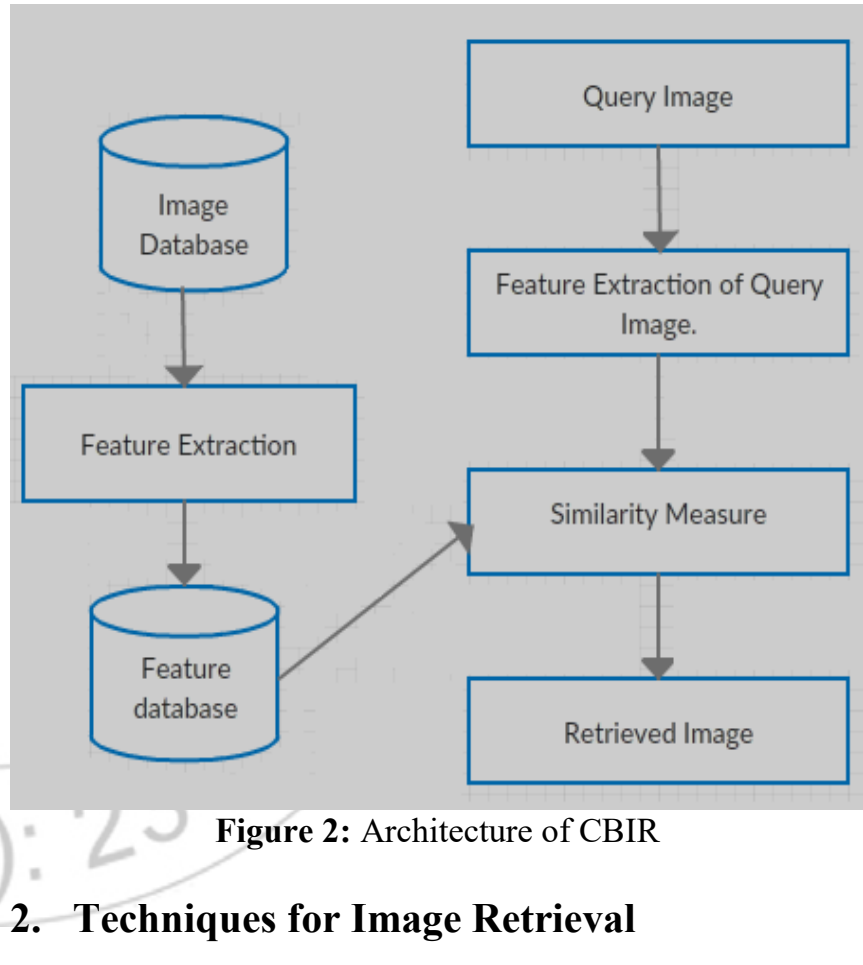

2.1 Kekre's transform applied on each row of Walsh transformed VQ codebook

This technique for image retrieval uses the color-texture features extracted from images based on vector quantization with Kekre's fast c odebook generation. 12 color descriptors per window (Red, Green and Blue per pixel) are obtained by dividing the database image into $2 \times 2$ pixel windows to form a vector. Then 16 code vectors are obtained from this set by applying Kekre's Fast Codebook Generation. The Walsh transform is applied on each column of the codebook, followed by Kekre' s transform applied on each row. This transformed vector is then used for image retrieval as the image signature. The method takes lesser computations as compared to conventional Walsh applied on complete image. The method gives the color-texture features of the image 


\section{International Journal of Science and Research (IJSR) \\ ISSN (Online): 2319-7064}

Index Copernicus Value (2015): 78.96 Impact Factor (2015): 6.391

database at reduced feature set size and gives better precision and recall as compared to full Walsh based CBIR. This method avoids resizing of images which is required for any transform based feature extraction method.

\subsection{Kekre's transform applied on each row of DCT transformed VQ codebook}

This technique uses the color-texture features extracted from images based on vector quantization with Kekre's fast codebook generation. 12 color descriptors per window (Red, Green and Blue per pixel) are obtained by dividing the database image into $2 \times 2$ pixel windows to form a vector. Then 16 code vectors are obtained from this set by applying Kekre's Fast Codebook Generation. The Discrete Cosine transform is applied on each column of the codebook, followed by Kekre's transform applied on each row. This transform vector then is used as the image signature for image retrieval. The method takes lesser computations as compared to conventional DCT applied on complete image [2]. The method gives the color-texture features of the image database at reduced feature set size and gives better precision and recall as compared to full DCT based CBIR. This method avoids resizing of images which is required for any transform based feature extraction method.

\subsection{Image retrieval based on indexing and relevance feedback}

Based on human perception based similarity measure, a new indexing scheme with relevance feedback can be used for image retrieval. This indexing scheme is designed based on the primary and secondary keys which are selected by analyzing the entropy of features. Mann-Whitney test is used to provide relevance feedback. The discriminating features from the relevant and irrelevant images in a retrieved set are identified by using Mann-Whitney test. Then emphasis of the discriminating features is updated to improve the retrieval performance. The indexing scheme gives faster result and relevance feedback improves performance. The disadvantage of this method is overhead of relevance feedback which will increase the response time.

\subsection{Image retrieval using image regions as query example}

In this technique, image regions are given as query example instead of whole image. Using Image region as query example has higher effectiveness than using whole image. Two approaches for combining shape features are used - an equal weight linear combination and classification using machine learning algorithm. The first one is simpler and at least as effective as using the machine learning algorithm. Color and shape features are used for retrieving similar regions. Single region query example is better than whole image query example. And multiple region query examples outperformed single region query example and whole image query example.

\subsection{Image retrieval using color-texture features extracted} from Walshlet Pyramid

In Walshlet Pyramid based image retrieval technique, the image features are extracted by applying Walshlets [6] on gray plane (average of red, green and blue) and color planes (red, green and blue components). The results show that precision and recall of Walshlets are better than complete Walsh based CBIR. Color-Walshlets based CBIR have greater precision and recall than Gray-Walshlets based CBIR. Higher level Walshlets give very coarse color-texture features. Hence Walshlets level-5 performs better retrieval than other Walshlets.

\subsection{Kekre transform over row mean, column mean using image tiling}

The process of dividing the image into non overlapping parts is called image tiling. A sample process of tiling is depicted in Fig.3. Kekre transform can be applied on row mean column mean and combination of both row mean and column mean on the image tiles. This is used as feature set for image retrieval. Experimental results shows that taking row mean, column mean and combination improves the performance of image retrieval as compared to taking Kekre Transform of full image[5].

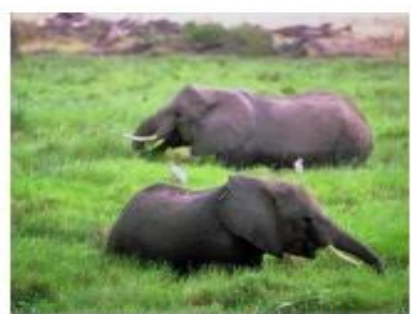

a. Full Image

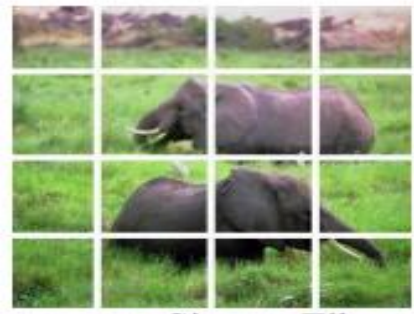

c. Sixteen Tiles

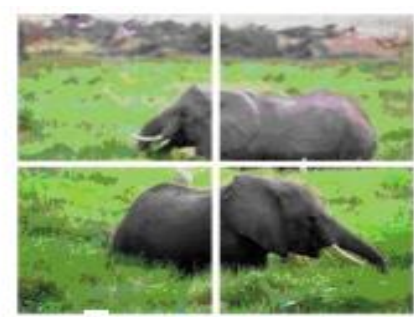

b. Four Tiles

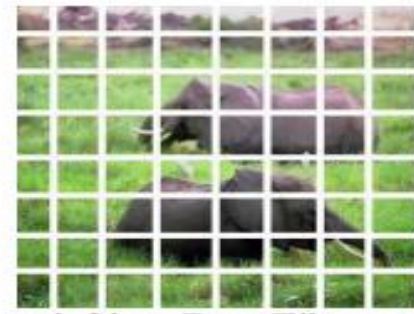

d. Sixty Four Tiles

\subsection{Localized content based image retrieval}

Localized Content-Based Image Retrieval is used when the user is interested in a portion of the image, and the rest of the image is irrelevant. A localized CBIR technique called Accio! uses labeled images in conjunction with a multipleinstance learning algorithm. Accio! first identifies the desired object. It then re-weights the features, and finally rank images in the database using a similarity measure. The similarity measure is based upon individual regions within the image. 


\subsection{CBIR using ACO-SVM with DTCWT and color} features

A new technique for CBIR by ant colony optimization (ACO) and Support vector machine (SVM) with dual tree complex wavelet transform (DTCWT) and color features was introduced in [8]. First extract texture feature using DTCWT. After that, extract color feature using dominant color descriptor (DCD) on RGB and HSV color space for improving computation and efficiency. The equal dimension is calculated using weighted Euclidean distance (WED). $\mathrm{ACO}$ is used for increasing the performance and data is classified using SVM.

\section{Conclusion}

This paper discussed some of the techniques adopted for Content based image retrieval. The choice of the technique is dependent upon the application, speed and expected accuracy of results. Such a study helps in exploring new ways to research in the area of image retrieval.

\section{References}

[1] H B Kekre, T K Sarode S D ,Thepade "Image Retrieval by Kekre's Transform Applied on Each Row of Walsh Transformed VQ Codebook", International Conference and Workshop on Emerging Trends in Technology, (ICWET 2010) - TCET, Mumbai,

[2] H B Kekre, T K Sarode S D , Thepade "Image Retrieval using Color-Texture Features from DCT on VQ Codevectors obtained by Kekre's Fast Codebook Generation”,CGST-GVIP Journal, Volume 9, Issue 5, September 2009.

[3] Amit K. Das , Bhabatosh Chanda ,Sanjoy K. Saha "Image retrieval based on indexing and relevance feedback", 2006 Elsevier B.V.

[4] D. N. F. Awang Iskandar, James A. Thom, S. M. M. Tahaghoghi "Content-based Image Retrieval Using Image Regions as Query Examples", Nineteenth Australasian Database Conference (ADC2008), Wollongong, Australia, January 2008.

[5] Dr. H.B.Kekre, Sudeep D. Thepade, Archana Athawale, Anant Shah, Prathamesh Verlekar and Suraj Shirke "Kekre Transform over Row Mean, Column Mean and Both Using Image Tiling for Image Retrieval',International Journal of Computer and Electrical Engineering, Vol.2, No.6, December, 2010

[6] H B Kekre, sudeep D Thepade "Image Retrieval using Color-Texture Features Extracted from Walshlet Pyramid",ICGST - GVIP Journal, ISSN: 1687-398X, Volume 10, Issue 1, February 2010

[7] Rouhollah Rahmani, Sally A. Goldman, Hui Zhang, John Krettek, and Jason E. Fritts "Localized Content Based Image Retrieval”, MIR'05, November 10-11, 2005, Singapore.

[8] Akash Gautama, Rinkoo Bhatiaa, "A Novel Method For CBIR Using ACO-SVM With DTCWT And Color Features", Science Direct, Materials today proceedings, 2016. 